\title{
Tomografia computadorizada de feixe cônico como exame complementar norteador em exodontia de terceiro molar semi-incluso e impactado próximo ao canal mandibular: relato de caso
}

\author{
Cone-beam computed tomography as a complementary guide exam in third-molar \\ semi included and impacted exodontia near the mandibular canal: case report
}

\author{
Tomografía computarizada de haz cónico como examen complementario de guía \\ en exodoncia de tercer molar semi retenido e impactado cerca del canal \\ mandibular: reporte de caso \\ Diego Filipe Bezerra SILVA ${ }^{1}$ \\ Danyllo Guimarães Morais BARROS ${ }^{2}$ \\ Jussara da Silva BARBOSA ${ }^{3}$ \\ Amaro Lafayette Nobre FORMIGA FILHO 4 \\ ${ }^{1}$ Graduado em Odontologia, Universidade Estadual da Paraíba - UEPB, 58429-600 Campina Grande-PB, Brasil \\ ${ }^{2}$ Graduando do Curso de Odontologia, Universidade Estadual da Paraíba-UEPB, 58429-600 Campina Grande-PB, Brasil \\ ${ }^{3}$ Mestranda do Programa de Pós-Graduação em Odontologia, Universidade Estadual da Paraíba-UEPB, \\ 58429-600 Campina Grande-PB, Brasil \\ ${ }^{4}$ Professor Doutor do Curso de Odontologia, Universidade Estadual da Paraíba - UEPB, 58429-600 Campina Grande-PB, Brasil
}

\section{Resumo}

Introdução: No contexto da cirurgia bucomaxilofacial, a exodontia de terceiros molares é um dos procedimentos mais comuns e diversas são as suas indicações. Sua realização pode causar diversas complicações pós-operatórias, como lesão no nervo alveolar inferior (NAI). Para evitar essas complicações, os exames de imagem pré-operatórios se tornam indispensáveis para um procedimento de sucesso. Objetivo: Apresentar, por meio de um relato de caso, a tomografia computadorizada de feixe cônico (TCFC) como exame complementar norteador em exodontia de terceiro molar semi-incluso e impactado próximo ao canal mandibular. Relato do caso: Paciente do sexo feminino, 20 anos de idade, apresentou-se para exodontia de terceiros molares com finalidade ortodôntica. $\mathrm{Na}$ análise radiográfica inicial, o dente 38 apresentava-se semi-incluso e impactado em mesio-inclinação, próximo ao dente 37 e com íntima relação com ocanal mandibular. A TCFC revelou que o trajeto do canal mandibular passava por vestibular das raízes do 38 e havia compartilhamento da cortical do canal com a cortical alveolar em ambas as raízes. Após as exodontias, foram feitas as suturas e prescrição de antibiótico e antiinflamatório, além de recomendações pós-operatórias para a paciente. Decorridos oito dias após a cirurgia, a paciente retornou para remoção das suturas, sem apresentar queixas em relação ao pós-operatório. Conclusão: O uso de exames de imagem, como a radiografia panorâmica e a TCFC, permitiu a visualização completa e precisa das estruturas anatômicas para que assim o procedimento fosse concluído sem implicações trans e pós-operatórias negativas.

Descritores: Cirurgia Bucal; Dente Impactado; Tomografia Computadorizada de Feixe Cônico.

\section{Abstract}

Introduction: In the context of maxillofacial surgery, the extraction of third molars is one of the most common procedures and several are their indications. Its performance can cause several postoperative complications, such as injury to the inferior alveolar nerve (IAN). To avoid such complications, preoperative imaging tests become indispensable for a successful procedure. Objective: To present, by means of a case report, cone-beam computed tomography (CBCT) as a complementary exam in third-molar third molar exodontia and impacted near the mandibular canal. Description of the clinical case: A 20-years-old female presented for extraction of third molars with orthodontic purpose. At initial radiographic analysis, tooth 38 was semi-included and impacted in mesio-inclination, close to tooth 37 and apparently within the mandibular canal. The CBCT revealed a relationship of proximity to the tooth 37. In addition, the mandibular canal pathway passed through the vestibular roots and the cortex was shared with the alveolar cortical in both roots. After the exodontia, the sutures and antibiotic and anti-inflammatory prescription were made, besides recommendations for the patient. Eight days after surgery, the patient returned for removal of the sutures, without presenting any complaints regarding the postoperative period. Conclusion: The use of imaging exams such as panoramic radiography and CBCT allowed the complete and accurate visualization of anatomical structures so that the procedure could be concluded without negative trans and postoperative implications.

Descriptors: Surgery, Oral; Tooth Impacted; Cone-Beam Computed Tomography.

\section{Resumen}

Introducción: En el contexto de la cirugía bucomaxilofacial, la exodoncia de terceros molares es uno de los procedimientos más comunes y diversos son sus indicaciones. Su realización puede causar diversas complicaciones postoperatorias, como lesión en el nervio alveolar inferior (NAI). Para evitar estas complicaciones, los exámenes de imagen preoperatorios se vuelven indispensables para un procedimiento de éxito. Objetivo: Presentar, por medio de un relato de caso, la tomografía computarizada de haz cónico (TCHC) como examen complementario orientador en exodoncia de tercer molar semi-incluido e impactado cerca del canal mandibular. Descripción del caso clínico: Paciente del sexo femenino, 20 años de edad, se presentó para exodoncia de terceros molares con finalidad ortodóntica. En el análisis radiográfico inicial, el diente 38 se presentaba semi-incluido e impactado en mesioinclinación, cerca del diente 37 y aparentemente dentro del canal mandibular. La TCHC reveló relación de proximidad con el diente 37. Además, el trayecto del canal mandibular pasaba por vestibular de las raíces y había compartido la cortical del canal con la cortical alveolar en ambas raíces. Después de las exodontias, se realizaron las suturas y prescripción de antibiótico y antiinflamatorio, además de recomendaciones para la paciente. Después de la cirugía, la paciente regresó para la remoción de las suturas, sin presentar quejas en relación al postoperatorio. Conclusión: El uso de exámenes de imagen, como la radiografía panorámica y la TCHC, permitieron la visualización completa y precisa de las estructuras anatómicas para que así el procedimiento fuera concluido sin implicaciones trans y postoperatorias negativas.

Descriptores: Cirugía Bucal; Diente Impactado; Tomografía Computarizada De Haz Cónico.

\section{INTRODUÇÃO}

No contexto da cirurgia bucomaxilofacial, a exodontia de terceiros molares é um dos procedimentos mais comuns, apresentando diversas indicações ${ }^{1-6}$. É um procedimento delicado, podendo causar diversas complicações pós-operatórias ${ }^{7,8}$. Dentre as mais frequentes destacam-se: tumefação, dor, hemorragia, trismo, alveolite e lesões no nervo alveolar inferior (NAI) ${ }^{1,3}$.

As lesões de caráter neurológico são graves e devem ser evitadas, porém são consideradas raras ${ }^{5}$, ocorrendo em cerca de $0,4 \%$ a $8 \%$ das cirurgias para remoção de terceiros 
molares $^{4,9}$. O dano ao NAI ocorre nesses casos por conta da relação espacial entre o terceiro molar e o canal mandibular, que revela uma proximidade muito acentuada entre a raiz do dente e o ramo do nervo ${ }^{1,3,5,7,9}$. Os danos podem ser causados por traumatismo direto ou indireto, pela compressão do nervo, pelo edema do local cirúrgico, ou até mesmo por hematoma pós-extração. Além disso, os danos podem ser transitórios, regredindo em semanas ou meses, porém partes desses distúrbios nervosos são permanentes e não são reparados ao longo do tempo ${ }^{7}$, ocorrendo em menos de $1,0 \%$ dos pacientes ${ }^{9}$.

É de extrema importância que o cirurgião-dentista esteja atento a essas condições e busque avaliar os riscos por meio de um planejamento cirúrgico adequado ${ }^{2,3,4,8}$. Para isso, os exames de imagem pré-operatórios se tornam indispensáveis para um procedimento bem sucedido ${ }^{5,6,7,9,10}$, destacando-se a radiografia panorâmica e a tomografia computadorizada de feixe cônico (TCFC) ${ }^{10}$. A TCFC é uma das mais novas técnicas de imagem utilizadas na Odontologia ${ }^{6}$, sendo um recurso imprescindível para obtenção de imagens claras necessárias para uma avaliação préoperatória adequada ${ }^{11}$.

Tendo em vista tais aspectos, o objetivo do presente estudo é apresentar, por meio de um relato de caso, a TCFC como exame complementar norteador no planejamento préoperatório em exodontia de terceiro molar semi-incluso e impactado próximo ao canal mandibular.

\section{CASO CLÍNICO}

Paciente do sexo feminino, 20 anos de idade, apresentou-se para exodontia de terceiros molares com finalidade ortodôntica. Para isto, uma radiografia panorâmica foi solicitada. $\mathrm{Na}$ análise radiográfica foi possível observar o dente 18 semi-incluso, o dente 28 semi-incluso e impactado e ausência do dente 48. Além disso, o dente 38 apresentava-se semi-incluso e impactado em mesioinclinação, íntima relação de proximidade com o dente 37 e com o canal mandibular (Figura 1). A TCFC, solicitada após necessidade de uma visualização tridimensional da região, revelou relação de proximidade com o dente 37, especialmente no terço cervical, mas não foram identificadas alterações deste. Em relação à proximidade das raízes do dente 38 com o canal mandibular, observou-se que o trajeto do canal passava por vestibular das raízes e havia compartilhamento da cortical do canal com a cortical alveolar em ambas as raízes (Figura 2). A paciente foi informada sobre o possível risco cirúrgico para o NAI e concordou em se submeter ao procedimento. As exodontias dos dentes 18, 28 e 38 foram feitas em uma única sessão (Figura 3). Foi realizada antissepsia bucal interna com clorexidina $0,12 \%$ e externa com clorexidina $2 \%$. Para exodontia dos dentes 18 e 28 , foram feitas anestesias terminais infiltrativas no fundo de sulco e por palatino, utilizando um tubete com 1,8 ml de anestésico lidocaína a $2 \%$ com epinefrina 1:200.000, para cada dente. Em seguida, foi realizada uma incisão relaxante com retalho em envelope para exposição dos dentes para posterior remoção dos mesmos com auxílio de uma alavanca Seldin reta. $\mathrm{Na}$ exodontia do dente 38 foi utilizado um tubete com o mesmo anestésico descrito anteriormente para anestesia do NAI pela técnica direta e, em seguida, foi utilizado mais um tubete para anestesia infiltrativa no fundo de sulco e por lingual. Após a paciente relatar sensação de dormência no lábio inferior, do lado esquerdo, iniciou-se o procedimento cirúrgico. Realizouse incisão relaxante com retalho em envelope para exposição da junção amelocementária do dente 38 , e com a broca $n^{\circ} 702$ em alta rotação e irrigação com soro fisiológico, foi realizada osteotomia nas faces vestibular e distal. Em seguida, foi feita odontossecção no sentido vestíbulo-lingual com broca cirúrgica Zekrya em alta rotação, para remover a porção mesial da coroa. Foi utilizada uma alavanca tipo Seldin curva para remover o dente do alvéolo. Após as exodontias, foram feitas as suturas e prescrição de amoxicilina $500 \mathrm{mg}$, por um período de sete dias, e nimesulida $100 \mathrm{mg}$ por três dias, além de recomendações pós-operatórias à paciente. A mesma, decorridos oito dias após a cirurgia, retornou para remoção das suturas, sem apresentar queixas.

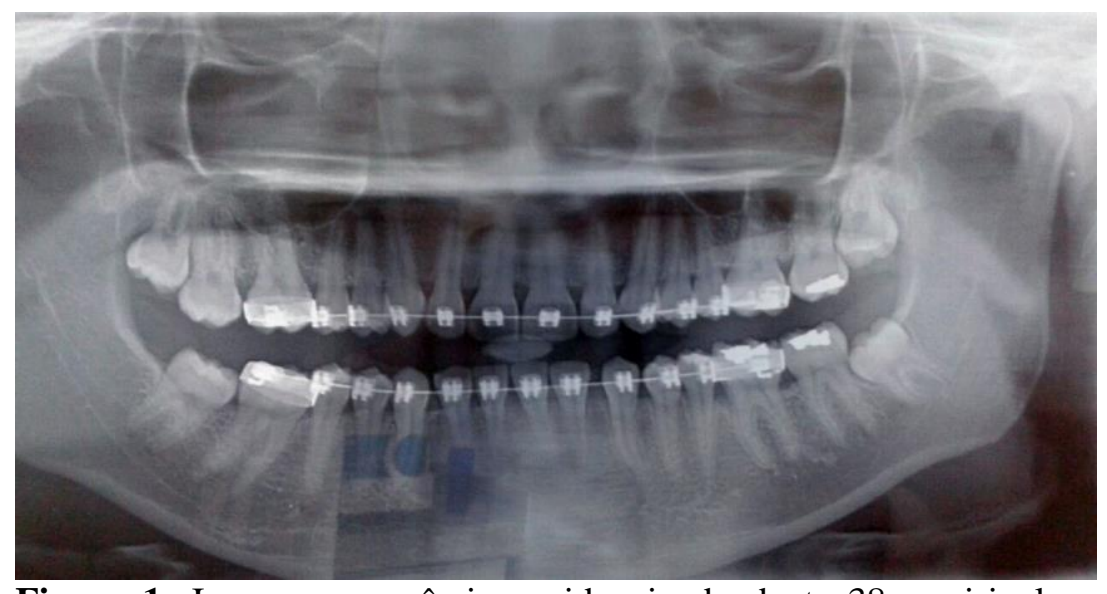

Figura 1: Imagem panorâmica evidenciando dente 38 semi-incluso com mesio-inclinação e íntima relação com o canal mandibular.

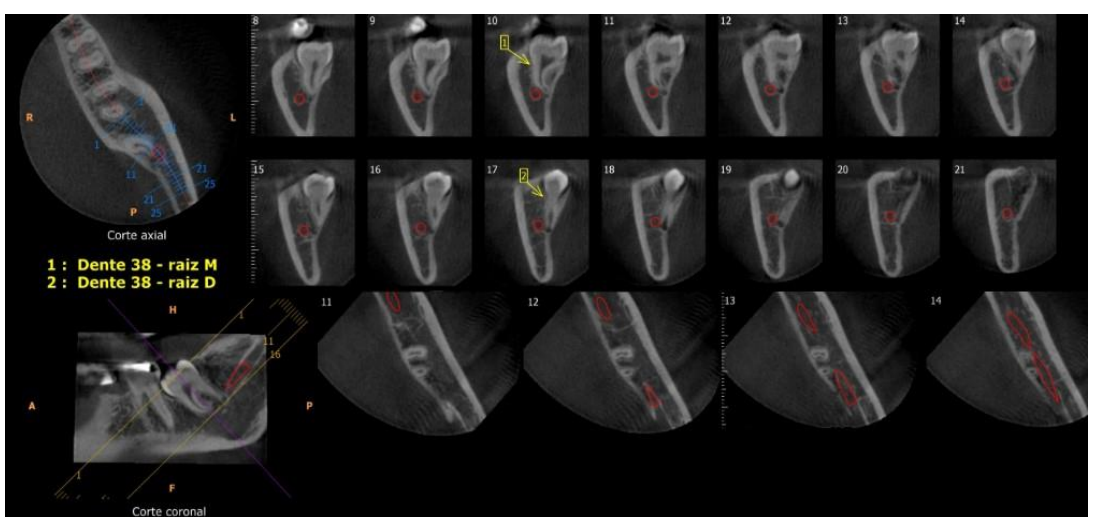

Figura 2: Tomografia computadorizada de feixe cônico nos cortes axial e coronal evidenciando o trajeto do canal mandibular passando por vestibular das raízes e compartilhamento da cortical do canal com a cortical alveolar em ambas as raízes.

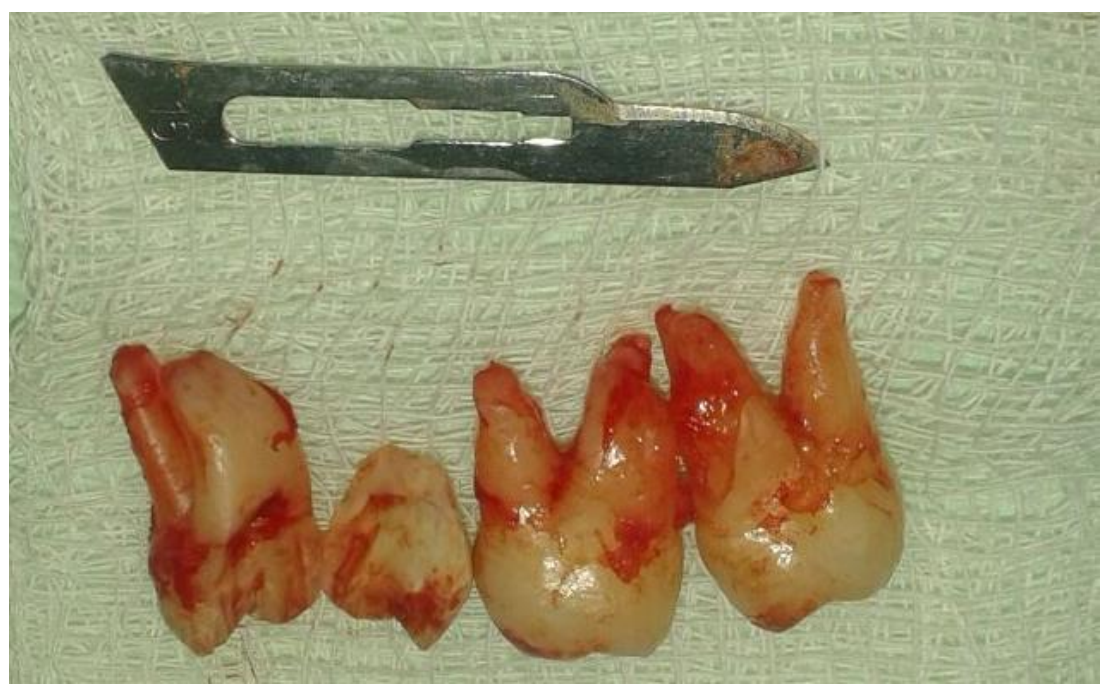

Figura 3: Dentes 18, 28 e 38 extraídos. Nota-se odontosecção do dente 38 .

\section{DISCUSSÃO}

A exodontia dos terceiros molares é um procedimento odontológico que requer indicação cirúrgica, planejamento e acompanhamento do paciente ${ }^{12}$. Uma de suas indicações é quando há espaço inadequado para sua erupção na região retromolar, entre o segundo molar e o ramo mandibular. Isso pode levar a uma erupção com implicações negativas do terceiro molar, criando uma aba de tecido gengival em torno do dente parcialmente erupcionado, ou bolsa pericoronal, potencialmente capaz de progredir para pericoronarite. A extração também é justificada quando há mau posicionamento mesioangular ou horizontal do terceiro molar, levando a dificuldades no controle de biofilme entre o segundo e o terceiro molares que ocasionalmente, pode promover o desenvolvimento de cáries dentárias de segundo molar ${ }^{10}$. No caso ora relatado, a paciente possuía o dente 38 mesioangulado e os dentes 18 e 28 necessitavam ser extraídos por questões ortodônticas, 
o que justificou a exodontia dos três dentes.

Apesar das indicações, as cirurgias de terceiros molares têm numerosas complicações, nas quais o deslocamento da raiz do dente ou do próprio dente são uma das mais perigosas ${ }^{6}$, e há alguns casos na literatura sobre essas ocorrências ${ }^{12,13}$. Outra complicação que pode ocorrer é a perfuração do seio maxilar, no caso dos terceiros molares superiores. A remoção do terceiro molar mandibular pode colocar os pacientes em risco de déficits neurossensoriais graves, particularmente devido à lesão no nervo lingual e no $\mathrm{NAI}^{10}$. Vários métodos imaginológicos estão disponíveis para o planejamento do tratamento antes da remoção dos terceiros molares mandibulares ${ }^{14}$ para avaliar o risco de lesão do NAI antes da operação ${ }^{5}$. As avaliações pré-operatórias de imagem tipicamente incluem a radiografia intraoral convencional ou radiografia panorâmica ${ }^{10}$.

Nas radiografias periapicais e radiografia panorâmica o canal mandibular pode ser visto como uma linha radiolúcida com duas bordas corticais finas. Algumas vezes as raízes se dobram distalmente ou mesialmente, o que poderia guiar o plano de operação e os cirurgiões-dentistas poderiam extrair as raízes seguindo a direção da curvatura. No entanto, quando as raízes se dobram vestibularmente ou lingualmente, a imagem radiográfica não é útil, pois sem visualização adequada, uma exodontia pode causar compressão ou laceração do $\mathrm{NAI}^{3}$. Além disso, na imagem, o dente ou suas raízes podem estar interpostos com o canal mandibular, como no caso ora relatado. Isso se deve ao fato de a radiografia panorâmica ser observada em apenas duas dimensões ${ }^{5,7}$. Recentemente, a TCFC tem sido amplamente utilizada no trabalho clínico devido à sua capacidade tridimensional ${ }^{5,6,7} \mathrm{e}$ torna-se o exame complementar ideal para avaliar a estreita relação entre os terceiros molares e o NAI ${ }^{10}$. Com o auxílio da TCFC, pode-se obter uma relação precisa do canal alveolar inferior e os ápices das raízes do dente, o que não pode ser feito por métodos radiográficos convencionais ${ }^{6}$.

Após a avaliação da imagem, o cirurgião-dentista será capaz de avaliar o risco de lesão ao NAI e de tomar a decisão sobre remover ou não o dente. No presente caso, optou-se pela exodontia, pois, apesar de as corticais das raízes dos dentes estarem intimamente relacionadas à cortical do canal mandibular, o NAI seguia vestibularmente em relação ao dente. Nos casos onde o NAI é ligado às raízes dos dentes, o risco de danos nervosos é ainda maior ${ }^{15}$.

\section{CONCLUSÃO}

No manejo cirúrgico de terceiros molares com proximidade acentuada ao canal mandibular, a prevenção de complicações neurológicas deve ser o principal objetivo do cirurgião-dentista. Para isso, um detalhado estudo do caso e um adequado planejamento operatório são indispensáveis, tendo em vista as morbidades que uma lesão pode causar. Nesse sentido, o presente relato de caso, destacou o uso de exames de imagem, como a radiografia panorâmica e a TCFC, que permitiram a visualização completa e precisa das estruturas anatômicas para que assim o procedimento fosse concluído sem implicações trans e pós-operatórias negativas.

\section{REFERÊNCIAS}

1. Camargo IB, Melo AR, Fernandes AV, Cunningham LL Jr, Laureano Filho JR, Van Sickels JE. Decision making in third molar surgery: a survey of Brazilian oral and maxillofacial surgeons. Int Dent J. 2015; 65(4):169-77.

2. Hasani A, Ahmadi Moshtaghin F, Roohi P, Rakhshan V. Diagnostic value of cone beam computed tomography and panoramic radiography in predicting mandibular nerve exposure during third molar surgery. Int $\mathrm{J}$ Oral Maxillofac Surg. 2017; 46(2):230-5.

3. Rodriguez Y, Baena R, Beltrami R, Tagliabo A, Rizzo S, Lupi SM. Differences between panoramic and Cone
Beam-CT in the surgical evaluation of lower third molars. J Clin Exp Dent. 2017; 9(2):e259-65.

4. Gu L, Zhu C, Chen K, Liu X, Tang Z. Anatomic study of the position of the mandibular canal and corresponding mandibular third molar on cone-beam computed tomography images. Surg Radiol Anat. 2018; 40(6):609-14

5. Mallick A, Vidya KC, Waran A, Rout SK. Measurement of lingual cortical plate thickness and lingual position of lower third molar roots using cone beam computed tomography. J Int Soc Prev Community Dent. 2017; 7(Suppl 1):S8-12.

6. Chen Y, Liu J, Pei J, Liu Y, Pan J. The risk factors that can increase possibility of mandibular canal wall damage in adult: a cone-beam computed tomography (CBCT) study in a Chinese population. Med Sci Monit. 2018; 24:26-36.

7. Huang TT, Chang CJ, Chen KC, Lo JB, Chen MY, Huang JS. Outcome analysis and unexpected-scenario prediction in 2-stage orthodontic lower third molar extraction. J Oral Maxillofac Surg. 2018; 76(3):503.e1-503.e8.

8. Ishii S, Abe S, Moro A, Yokomizo N, Kobayashi Y. The horizontal inclination angle is associated with the risk of inferior alveolar nerve injury during the extraction of mandibular third molars. Int J Oral Maxillofac Surg. 2017; 46(12):1626-34.

9. Korkmaz YT, Kayımaz S, Senel FC, Atasoy KT, Gumrukcu Z. Does additional cone beam computed tomography decrease the risk of inferior alveolar nerve injury in high-risk cases undergoing third molar surgery? Does CBCT decrease the risk of IAN injury? Int J Oral Maxillofac Surg. 2017; 46(5):628-35.

10. Lee B, Park Y, Ahn J, Chun J, Park S, Kim M et al. Assessment of the proximity between the mandibular third molar and inferior alveolar canal using preoperative 3D-CT to prevent inferior alveolar nerve damage. Maxillofac Plast Reconstr Surg. 2015; 37(1):30

11. Nakamori K, Tomihara K, Noguchi M. Clinical significance of computed tomography assessment for third molar surgery. World J Radiol. 2014; 6(7):417-23.

12. Silveira RJ, Garcia RR, Botelho TL, Franco A, Silva RF. Accidental displacement of third molar into the sublingual space: a case report. J Oral Maxillofac Res. 2014; 5(3):e5.

13. Kamburoglu K, Kursun S, Oztas B. Submandibular displacement of a mandibular third molar root during extraction: a case report. Cases J. 2010; 3:8.

14. Matzen LH, Christensen J, Hintze H, Schou S, Wenzel A. Influence of cone beam CT on treatment plan before surgical intervention of mandibular third molars and impact of radiographic factors on deciding on coronectomy vs surgical removal. Dentomaxillofac Radiol. 2013; 42(1):98870341.

15. Peker I, Sarikir C, Alkurt MT, Zor ZF. Panoramic radiography and cone-beam computed tomography findings in preoperative examination of impacted mandibular third molars. BMC Oral Health. 2014; 14:71.

\section{CONFLITO DE INTERESSES}

Os autores declaram não haver conflitos de interesse.

\section{AUTOR PARA CORRESPONDÊNCIA}

\section{Amaro Lafayette Nobre Formiga Filho}

amarolafayette@gmail.com

Submetido em 10/03/2018 Aceito em 11/05/2018 